displacement field showcases twisted bilayer $\mathrm{WSe}_{2}$ as a good material platform to study the interplay between interactions and frustration. Compared to graphite systems, the single-particle bands here are spin-polarized, and the system is in the moderate correlated regime, which may give rise to different physics, certainly worthy of future exploration.

\section{Junwei Liu (D)}

Department of Physics, Hong Kong University of Science and Technology, Clear Water Bay, Hong Kong, China.

凶e-mail:liuj@ust.hk

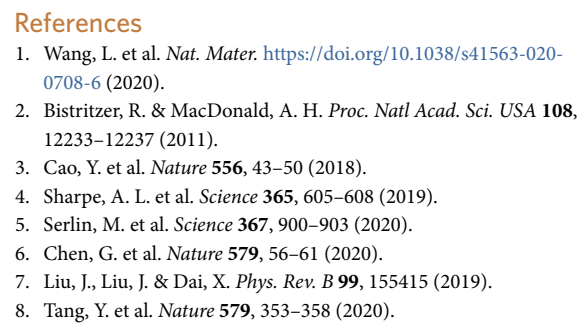

\title{
Long-acting antiretroviral therapy
}

\author{
Nanoformulations of cabotegravir prodrugs have been developed to prolong the pharmacokinetics of the \\ antiretroviral agent. They can be present in the plasma of mice and non-human primates for a year following \\ a single injection.
}

\section{Vicente Soriano, Pablo Barreiro and Carmen de Mendoza}

T he fight against viruses with epidemic proportions largely relies on antiviral agents and vaccines. Depending on the ability of a given virus to produce persistent infection, antiviral agents are designed to shorten and ameliorate symptoms, eradicate infection, or suppress viral replication and prevent disease progression. To suppress viral replication, well exemplified by the treatments for human immunodeficiency virus (HIV) or hepatitis B, antiviral therapy has to be administered for the lifetime of the patient, otherwise viral rebound occurs and the disease resumes. Vaccines have been developed with great success for many viral infections, including measles, polio and rubella. However, smallpox is the only one to be eradicated in humans - almost half a century ago following massive vaccine campaigns. Unfortunately, vaccines have remained elusive for certain pathogenic viruses, such as HIV, with antiretroviral therapy (ART) being the only option available to confront such a disease ${ }^{1}$.

Since the advent of antiretroviral drugs in the 1990s, the prognosis of HIV-infected individuals has drastically improved. Indeed, under good adherence to medications, immunological damage no longer occurs, and the life expectancy of HIV-positive individuals taking ARTs has approached that of the HIV-negative population ${ }^{2}$. As a result, AIDS-defining illnesses are rarely seen nowadays, but instead in individuals who are unaware of their infection presenting late symptoms in clinics. Improvements made to ARTs during the past 25 years have been tremendous. Current ARTs are often in the form of an oral pill prescribed once a day. However, further efforts have resulted in the recent development of long-acting antiretroviral drugs, such as cabotegravir and rilpivirine, that are administered by injection intramuscularly on a monthly basis ${ }^{3,4}$. In the clinic, these formulations are being considered for both maintenance therapy in patients already under conventional daily oral ART as well as for pre-exposure prophylaxis (PrEP) in HIV-negative vulnerable individuals. However, these medications may produce local injection site reactions, may be associated with harmful drug interactions, and require monthly visits to clinics ${ }^{5,6}$.

Writing in Nature Materials, Tanmay Kulkarni and colleagues ${ }^{7}$ report the development of long-acting cabotegravir (CAB) prodrug nanocrystals with the ability to prolong the pharmacokinetics of this antiretroviral agent by extending its presence in the blood for a year following a single intramuscular injection. The authors created poloxamer-coated CAB nanocrystal prodrugs with 14,18 and 22 carbon chains that were encased in biocompatible surfactants. These formulations were then tested for drug release, activation, cytotoxicity, antiretroviral inhibitory effect and biodistribution. Pharmacokinetic studies were subsequently performed in mice and rhesus macaques, with the lead 18-carbon ester chain NM2CAB displaying the most appealing profile (Fig. 1). Of note, no adverse events were recorded in the animal studies.

Kulkarni and colleagues demonstrated that the NM2CAB prodrug, administered as a single intramuscular injection, generated plasma $\mathrm{CAB}$ concentrations above the protein-adjusted $90 \%$ inhibitory concentration for up to one year in mice and also slow plasma CAB decay kinetics evident in rhesus macaques for up to 364 days. The medication exhibited unique properties in terms of prolonged drug release due to selective macrophage drug uptake and wide biodistribution across different organ tissues, including lymphoid, mucosal, gut and brain. Given the nanocrystal formulation, volumes of less than one millilitre would be needed for yearly administration in humans, reducing the likelihood of local injection site reactions. Furthermore, the authors indicated that a further advantage of NM2CAB nanocrystal fabrication is in the easy scalability since their methodology is conventionally employed in the pharmaceutical industry for increasing the purity of the active molecule.

There are several advantages of having year-long antiretrovirals when compared to month-long therapies. They would address important challenges such as injection site reactions and the need to attend monthly visits to clinics. Vulnerable populations in resource-rich countries and individuals in developing regions for whom regular attendance at healthcare sites is very difficult or beyond their financial means would benefit from year-long therapies. Furthermore, individuals infected with HIV-2 instead of HIV-1 or those dually infected with HIV-1 and HIV-2 may benefit alike, since $\mathrm{CAB}$ is active against both HIV-1 and HIV- $2^{8}$.

A number of caveats must be considered before translation of these year-long NM2CAB drugs towards broad clinical use. 
a
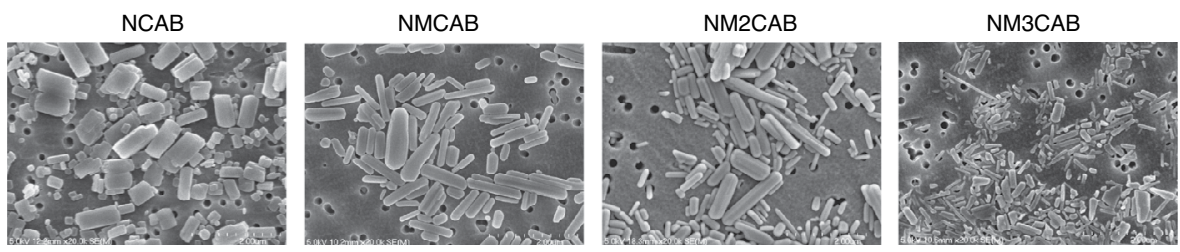

b

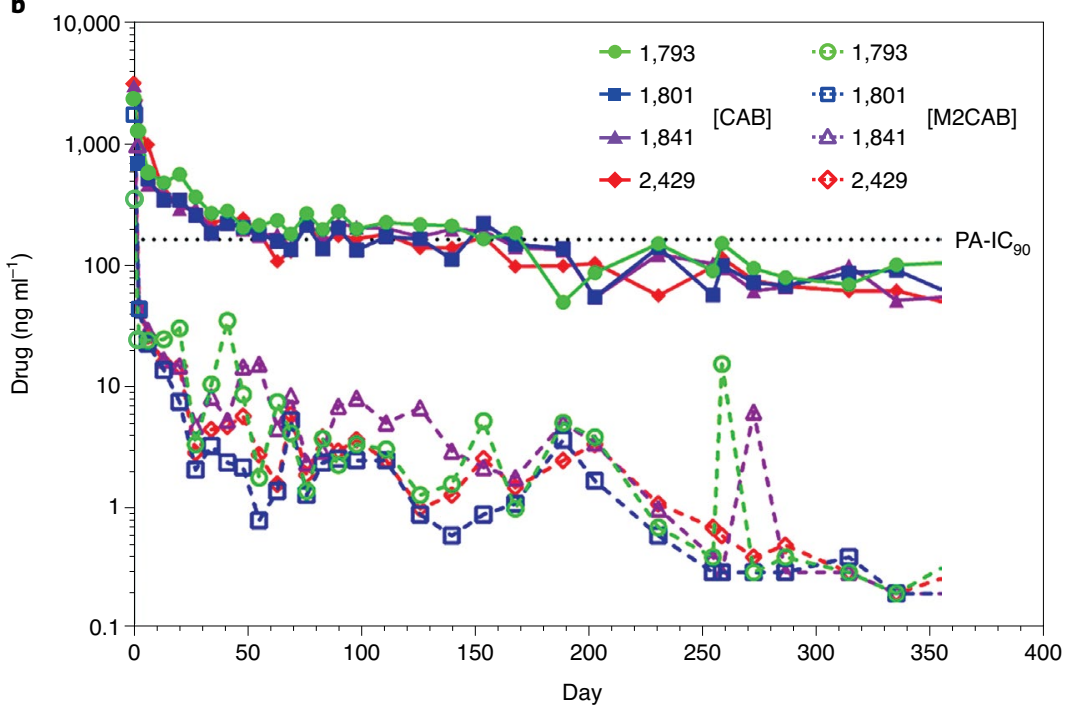

Fig. 1 | Cabotegravir prodrug nanoformulations and their release in vivo. a, Scanning electron microscopy images of cabotegravir prodrug nanoformulations and modifications done by attaching fatty acid esters of variable carbon lengths (NMCAB, NM2CAB and NM3CAB). b. Plasma CAB and M2CAB prodrug levels measured for 365 days following a single intramuscular injection in rhesus macaques. Figure adapted with permission from ref. ${ }^{7}$, Springer Nature Ltd.

Most importantly, the potential for drug interactions with other medications must be kept in mind using such a long-term dosage forms, to avoid potentially harmful pharmacokinetic side-effects. Second, the combination of multiple ARTs could be critical for the effective treatment of HIV infections. In the meantime, an appealing indication for a medication such as

NM2CAB will be as PrEP treatment for prevention of HIV in vulnerable individuals at high risk of contracting the infection. In a recent phase- $2 \mathrm{~b} / 3$ clinical trial by the HIV Prevention Trials Network (HPTN 083), intramuscular injection of long-acting cabotegravir (ViiV Healthcare) into vulnerable individuals every two months led to lower incidence of HIV infection than daily oral treatment (Truvada, Gilead Sciences ${ }^{9}$ ). Overall, very long-acting therapeutics such as these nanocrystal formulations could act as ideal medication in the absence of an HIV vaccine and may also be an equally effective approach for the development of therapeutic agents for other viral diseases such as COVID-19 ${ }^{10}$.

Vicente Soriano (D) $1,2 \times$, Pablo Barreiro ${ }^{3}$ and Carmen de Mendoza ${ }^{4}$

${ }^{1}$ UNIR Medical Center, Madrid, Spain. ${ }^{2}$ UNIR Health Sciences School, Madrid, Spain. ${ }^{3}$ Hospital Carlos III-La Paz, Madrid, Spain. ${ }^{4}$ Puerta de Hierro University Hospital, Majadahonda, Spain.

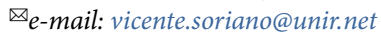

Published online: 23 July 2020 https://doi.org/10.1038/s41563-020-0731-7

References

1. Saag, M. S. et al. JAMA 320, 379-396 (2018).

2. Gueler, A. et al. AIDS 31, 427-436 (2017).

3. Swindells, S. et al. N. Engl. J. Med. 382, 1112-1123 (2020)

4. Orkin, C. et al. N. Engl. J. Med. 382, 1124-1135 (2020).

5. Benítez-Gutiérrez, L. et al. Expert Rev. Clin. Pharmacol. 11, 507-517 (2018).

6. Currier, J. S. N. Engl. J. Med. 382, 1164-1165 (2020).

7. Kulkarni, T. A. et al. Nat. Mater. https://doi.org/10.1038/s41563020-0674-z (2020).

8. Requena, S. et al. AIDS 33, 2167-2172 (2019).

9. Cohen, J. Science https://doi.org/10.1126/science.abc8634 (2020).

10. Beigel, J. H. et al. N. Engl. J. Med. https://doi.org/10.1056/ NEJMoa2007764 (2020).

\section{ELECTRON MICROSCOPY}

Check for updates

\section{A 3D map of atoms in 2D materials}

Scanning atomic electron tomography measurements reveal the 3D local structure around single dopant atoms in 2D transition metal dichalcogenides, providing essential information to investigate and predict their electronic properties.

\section{Angus I. Kirkland}

$\mathrm{R}$ eliable deployment in applications of graphene and other two-dimensional (2D) materials requires a complete understanding - at the atomic level - of the nature of defects that can significantly affect their physical properties. In particular, point defects, dislocations and dopant atoms are almost inevitably present in all as-synthesized materials, including graphene $^{1}$, hexagonal boron nitride ${ }^{2}$ and 2D transition metal dichalcogenides ${ }^{3}$. However, with only limited exceptions ${ }^{4}$, prior studies have mostly provided structural information projected on $2 \mathrm{D}$ images, whereas, to fully understand the structure and consequential effects of these defects, it is necessary to probe the full three-dimensional (3D) atomic map of the investigated materials.

Now, writing in Nature Materials, an interdisciplinary team lead by John Miao ${ }^{5}$ has used aberration-corrected scanning 